\title{
The Intrinsic Differences Between Immune Checkpoint Inhibitor Induced Myocarditis: A Retrospective Analysis of Real-World Data
}

\section{Yanna Lei}

West China Hospital of Sichuan University

\section{Xiufeng Zheng}

West China Hospital of Sichuan University

Qian Huang

West China Hospital of Sichuan University

Xiaoying Li

West China Hospital of Sichuan University

Meng Qiu

West China Hospital of Sichuan University

Ming Liu ( $\nabla$ liuming629@wchscu.cn )

West China Hospital of Sichuan University

\section{Research Article}

Keywords: Immune checkpoint inhibitors, Immune-related myocarditis, Immune-related myositis, Troponin, Lactate dehydrogenase

Posted Date: January 6th, 2022

DOI: https://doi.org/10.21203/rs.3.rs-1192888/v1

License: (c) (1) This work is licensed under a Creative Commons Attribution 4.0 International License. Read Full License 


\section{Abstract}

Immune-related myocarditis is a severe and even life-threatening immune-related adverse event (irAE) which may also be under-estimated due to the challenge in diagnosis. There have been few reports about the intrinsic difference between patients with immune-associated myocarditis. A retrospective analysis was conducted between March 2019 to June 2020 in West China Hospital and 18 patients with immunerelated myocarditis were studied. Cases were classified as mild $(n=12)$ or severe myocarditis $(n=6)$ according to the clinical manifestations and haemodynamic complications. Covariates extracted from medical records were compared in different groups, and factors associated with severe myocarditis were identified. In this retrospective analysis, the median age of the 18 patients was 60 years old. Most cases occur early and approximately after the first or second ICI infusion. The severity of myocarditis may be correlated with lactate dehydrogenase $(\mathrm{LDH})(P=0.04)$ and troponin $(P=0.0057)$. The relationship between troponin and myocarditis was further confirmed in another cohort which including 30 patients. In addition, patients are more likely to develop multi-irAEs, and myositis was the most common second irAE. Those who experience multi-irAEs had significantly higher $\mathrm{LDH}(\mathrm{P}=0.02)$ as well as myoglobin levels $(P=0.02)$ than did not experience. All patients were treated with steroids timely and the mortality rate was $5.6 \%$ in our study. In this study, we explore risk factors for severe myocarditis and we emphasized the importance of a multidisciplinary team in assisting diagnosis and treatment options. It is critical to initiate corticosteroid therapy, regardless of the severity of the myocarditis.

\section{Introduction}

The advent of immune-checkpoint inhibitors (ICls) which blocking cytotoxic T lymphocyte associated protein-4 (CTLA-4) and the programmed death-1 (PD-1)/programmed death ligand-1 (PD-L1) axis, both as monotherapy or in combination strategies, have revolutionized cancer treatment ${ }^{1-3}$. However, as the use of ICls in clinical practice has increased, immune-mediated toxicities have been observed, which can impact numerous organ systems, including the heart ${ }^{4,5}$. Immune-related myocarditis is the most serious life-threatening toxicity and the underlying pathomechanism is poorly understood ${ }^{6}$. The incidence of immune-related myocarditis caused by $\mathrm{ICl}$ is approximately $1 \%$, but the mortality rate can be as high as $46 \% 7,8$. Because of its rarity, information on this unusual disease is limited, and further research is needed to fully understand the devastating irAEs. Furthermore, there is a scarcity of patients' data on the clinical characteristics, results of pertinent examinations, and outcomes of ICl-related cardiotoxicity.

To gain a better knowledge of this disease, we analyzed and described the characteristics of patients with immune-related myocarditis, including baseline clinical characteristics, drug-related data, relevant examination results, and outcomes. Our research will look into the internal disparities between patients.

\section{Materials And Methods \\ 2.1 Study population}


In this single-center retrospective study, data were collected from 18 patients with ICl-related myocarditis in West China Hospital from March 2019 to June 2020. Inclusion criteria were listed as follows: (1) a histologically confirmed diagnosis of solid malignancy in West China Hospital;(2) age $\geq 18$ years at ICI initiation; (3) received at least one cycle of $\mathrm{ICl}$;(4) met the diagnostic criteria through multidisciplinary panel decision;(5) exclude the possible diagnosis of coronary artery disease and myocarditis caused by other reasons. The study was approved by the institutional review board, and the requirement for written informed consent was waived. The present study was approved by the institutional review board of West China Hospital and was exempted from informed consent requirements owing to its retrospective design.

This article does not contain any studies with human participants or animals performed by any of the authors. All methods were performed in accordance with the relevant guidelines and regulations.

\subsection{Patients data collection}

The following characteristics of patients were recorded: (1) clinical baseline characteristics: age, gender, smoking and alcohol status, comorbid conditions, disease stage, cancer type and histological subtype et.al; (2) ICl-related information: type of ICl, treatment start date, the date of diagnosis, therapy line, combination with other drugs, response patterns; (3) relevant examination results: laboratory data including myocardial markers and infection-related indicators, echocardiography, coronary angiography, coronary computed tomography (CT) et.al; (4) treatment and outcome.

\subsection{Statistical Analysis}

Study variables were presented as number ( $\mathrm{n})$ - percentage (\%) and mean \pm standard deviation. Wilcoxon rank-sum tests are used for continuous variables and $\chi 2$ or Fisher's exact test is used for categorical variables. Missing data will not be analyzed in this study. The statistical analyses were conducted via R 4.0.3 and GraphPad Prism 8. P $<0.5$ was a statistically significant standard.

\section{Results}

\subsection{Baseline Characteristics}

Among the 18 patients who met the selection criteria were retrospectively identified in this study, and the patients were classified into mild $(n=12)$ and severe myocarditis $(n=6)$ according to the clinical manifestation and whether they had haemodynamic complications such as heart failure, cardiogenic shock, or arrhythmia. The clinical characteristics of the 18 patients with immune-related myocarditis are shown in Table 1. 
Table 1

Clinical characteristics of the patients and information of $\mathrm{ICl}$ in patients with immune-related myocarditis.

$\begin{array}{lll}\text { Mild group } & \text { Severe group } & \text { All Cases } \\ (\mathrm{N}=12) & (\mathrm{N}=6) & (\mathrm{N}=18)\end{array}$

Age(years)

0.08

Median [Min, Max]

61.5

44.5

60.0

$[45.0,79.0]$

$[29.0,67.0]$

$[29.0,79.0]$

\section{Gender}

Male

10 (83.3\%)

5 (83.3\%)

$15(83.3 \%)$

Female

$2(16.7 \%)$

1 (16.7\%)

$3(16.7 \%)$

Smoking history

Yes

5 (41.7\%)

$1(16.7 \%)$

$6(33.3 \%)$

No

7 (58.3\%)

5 (83.3\%)

$12(66.7 \%)$

Alcohol history

Yes

$2(16.7 \%)$

1 (16.7\%)

3 (16.7\%)

No

10(83.3\%)

5 (83.3\%)

15(83.3\%)

\section{Diabetes}

Yes

3 (25.0\%)

2 (33.3\%)

$5(27.8 \%)$

No

9 (75.0\%)

4 (66.7\%)

$13(72.2 \%)$

Hypertension

Yes

7 (58.3\%)

$0(0 \%)$

7 (38.9\%)

No

$5(41.7 \%)$

$6(100 \%)$

11 (61.1\%)

\section{Cancer type}

lung cancer

$9(75.0 \%)$

1 (16.7\%)

$10(55.6 \%)$

thymic carcinoma

Other cancers

1 (8.3\%)

3 (50.0\%)

4 (22.2\%)

2(16.7\%)

2 (33.3\%)

$4(22.2 \%)$

Cancer stage

Stage IV

$8(66.7 \%)$

$5(83.3 \%)$

0.24

0.048

0.04

ICl, immune checkpoint inhibitor; PD, progressive disease; PD-L1, programmed death-ligand 1; PR, partial response, $S D$, stable disease; $P D$, progressive disease. Tumour responses were assessed using computer tomography and determined using RECIST version 1.1. 


\begin{tabular}{|c|c|c|c|c|}
\hline & $\begin{array}{l}\text { Mild group } \\
(\mathrm{N}=12)\end{array}$ & $\begin{array}{l}\text { Severe group } \\
(\mathrm{N}=6)\end{array}$ & $\begin{array}{l}\text { All Cases } \\
(\mathrm{N}=18)\end{array}$ & $\mathbf{P}$ \\
\hline Stage III & $4(33.3 \%)$ & $1(16.7 \%)$ & $5(27.8 \%)$ & \\
\hline Clinical symptoms & & & & 0.141 \\
\hline Yes & $3(25.0 \%)$ & $6(100 \%)$ & $9(50 \%)$ & \\
\hline No & $9(75.0 \%)$ & $0(0 \%)$ & $9(50 \%)$ & \\
\hline Initial treatment line & & & & 0.01 \\
\hline 1 st & $9(75.0 \%)$ & $2(33.3 \%)$ & $11(61.1 \%)$ & \\
\hline $2 n d$ & $2(16.7 \%)$ & $0(0 \%)$ & $2(11.1 \%)$ & \\
\hline$\geq 3 \mathrm{rd}$ & $0(0 \%)$ & $4(66.7)$ & $4(22.2 \%)$ & \\
\hline Missing & $1(8.3 \%)$ & $0(0 \%)$ & $1(5.6 \%)$ & \\
\hline ICl type & & & & 1.00 \\
\hline Anti-PD-1 & $10(83.3 \%)$ & $5(83.3 \%)$ & $15(83.3 \%)$ & \\
\hline Anti-PD-L1 & $2(16.7 \%)$ & $1(16.7 \%)$ & $3(16.7 \%)$ & \\
\hline Treatment category & & & & 0.42 \\
\hline $\mathrm{ICl}$ alone & $6(50 \%)$ & $3(50.0 \%)$ & $9(50.0 \%)$ & \\
\hline Chemo $+\mathrm{ICl}$ & $6(50 \%)$ & $2(33.3 \%)$ & $8(44.4 \%)$ & \\
\hline Targeted + ICI & $0(0 \%)$ & $1(16.7 \%)$ & $1(5.6 \%)$ & \\
\hline Times to onset & & & & 0.40 \\
\hline Mean (SD) & $64.8(52.3)$ & $36.2(20.6)$ & $55.2(45.7)$ & \\
\hline Median [Min, Max] & $57.0[4.00,155]$ & $32.5[14.0,69.0]$ & $51.0[4.00,155]$ & \\
\hline Number of ICl cycles & & & & 0.38 \\
\hline 1 & $4(33.3 \%)$ & $3(50.0 \%)$ & $7(38.9 \%)$ & \\
\hline 2 & $4(33.3 \%)$ & $2(33.3 \%)$ & 6(33.3\%) & \\
\hline$\geq 3$ & $4(33.3 \%)$ & $1(16.7 \%)$ & $5(27.8 \%)$ & \\
\hline Mean (SD) & $2.75(2.22)$ & $1.67(0.82)$ & 2.39(1.91) & \\
\hline Median [Min, Max] & $2.00[1.00,8.00]$ & $1.50[1.00,3.00]$ & $2.00[1.00,8.00]$ & \\
\hline
\end{tabular}

$\mathrm{ICl}$, immune checkpoint inhibitor; PD, progressive disease; PD-L1, programmed death-ligand 1; PR, partial response, $S D$, stable disease; $P D$, progressive disease. Tumour responses were assessed using computer tomography and determined using RECIST version 1.1. 


\begin{tabular}{|lllll|}
\hline & $\begin{array}{l}\text { Mild group } \\
(\mathbf{N}=12)\end{array}$ & $\begin{array}{l}\text { Severe group } \\
(\mathbf{N}=6)\end{array}$ & \multicolumn{1}{|c|}{$\begin{array}{l}\text { All Cases } \\
\mathbf{( N = 1 8 )}\end{array}$} & 0.299 \\
\hline Efficacy of ICls & & & P \\
\hline PR & $5(41.7 \%)$ & $0(0 \%)$ & $10(55.6 \%)$ \\
\hline SD & $6(50.0 \%)$ & $4(66.7 \%)$ & $1(5.6 \%)$ \\
\hline PD & $1(8.3 \%)$ & $0(0 \%)$ & $2(11.1 \%)$ \\
\hline Missing & $0(0 \%)$ & $2(33.3 \%)$ & \\
\hline $\begin{array}{l}\text { ICl, immune checkpoint inhibitor; PD, progressive disease; PD-L1, programmed death-ligand 1; PR, } \\
\text { partial response, SD, stable disease; PD, progressive disease. Tumour responses were assessed using } \\
\text { computer tomography and determined using RECIST version 1.1. }\end{array}$ \\
\hline
\end{tabular}

The median age among the 18 patients was 60 years old (range from 29.0 to 79.0 years), $83.3 \%$ were male, and a history of smoking was reported in $33.3 \%$. Alcohol consumption was reported in $16.7 \%$ of the patients. $27.8 \%$ of patients had a history of diabetes and $38.9 \%$ of patients had hypertension. Lung cancer was the most common cancer diagnosis (55.6\%) and thymic carcinoma (22.2\%), followed by bladder cancer (5.56\%), sigmoid colon cancer (5.56\%), head and neck cancer (5.56\%) (Figure 1). In addition, the majority of patients $(72.2 \%)$ were in stage IV cancer at the time of ICl commencement. Half of the patients were asymptomatic. The most common clinical manifestations included dyspnea and fatigue.

In the mild myocarditis group, the mean age was 63.3 years old (range from 45.0 to 79.0 years) and in the severe group was 46.8 years old (range from 29.0 to 67.0 years). Lung cancer was the most prevalent cancer type in the mild group (75.0\%), but thymic carcinoma was the most common cancer type in the younger age group (50.0\%). Furthermore, none of the patients with severe myocarditis had a history of hypertension.

\subsection{Treatment-related Characteristics}

The majority of patients (61.1\%) received ICls as first-line therapy, while chemotherapy, not immunotherapy, has been used as first- or second-line therapy in the severe myocarditis group. In $66.7 \%$ of patients with severe myocarditis, ICls have been used as a third-line or later treatment (Table 1 ). According to the distinct mechanisms of action of ICls, 15 patients (83.3\%) received anti-PD-1 therapy and the remaining 3 patients (16.7\%) received anti-PD-L1 treatment. The percentage distribution of 18 cases was as follows: Pembrolizumab 33.3\%, Sintilimab 22.2\%, Camrelizumab 16.7\%, and other ICls $27.8 \%$, according to various $\mathrm{ICl}$ administered.

The median cycles of the onset of immune-related myocarditis from the initiation of ICI infusion were 2 (range, 1-8 cycles), and the median time was 51.0 days (range, 4-155 days). The number of days from the initiation of $\mathrm{ICl}$ infusion to the presentation of myocarditis in the mild group was longer than in the severe 
group. The mean time was 64.8 and 36.2 days in the mild verse severe group, respectively. Approximately $72.2 \%$ of patients developed myocarditis after 1 or $2 \mathrm{ICl}$ infusion cycles. For patients with severe myocarditis, only one patient received more than 3 cycles at the time of diagnosis. Tumour responses were assessed using computer tomography and determined using the RECIST version 1.1. Overall, 5 patients experienced partial responses, 10 patients exhibited stable disease, and 1 patient had progressive disease after $\mathrm{ICl}$ administration.

Patients were divided into three groups based on their treatment regimen. The proportion of participants who used $\mathrm{ICl}$ alone, chemotherapy $+\mathrm{ICl}$, and targeted therapy $+\mathrm{ICl}$ were $50.0 \%, 44.4 \%$, and $5.60 \%$, respectively. Due to the small number of participants in this study, people treated with $\mathrm{ICl}$ alone $(\mathrm{N}=9)$ and Chemo+ICl $(\mathrm{N}=8)$ will be discussed further (Table 2). The median onset time was earlier in the Chemo + $\mathrm{ICl}$ group compared with ICl alone cohort (34.5 v.s 69.0 days). In mild-myocarditis group, the onset time of $\mathrm{ICl}$ alone was 109 days, which was longer than patients who received Chemo + ICl (onset time:34.5 days). Timing of the development of immune-related myocarditis with a mean delay of 78.9 days and a median of 2 doses from the first $\mathrm{ICl}$ administration in the monotherapy group. Details of other information in each subgroup are listed in the Table 2. 
Table 2

Comparison of treatment regimens in myocarditis patients.

\begin{tabular}{|c|c|c|c|}
\hline & $\mathrm{ICl}$ alone $(\mathrm{N}=9)$ & $\mathrm{ICl}+$ Chemo $(\mathrm{N}=8)$ & $P$ value \\
\hline Age & & & 0.96 \\
\hline Median [Min, Max] & $60.0[33.0,79.0]$ & $61.5[29.0,75.0]$ & \\
\hline Cancer type & & & 0.08 \\
\hline lung cancer & $3(33.3 \%)$ & $7(87.5 \%)$ & \\
\hline thymic carcinoma & $3(33.3 \%)$ & $1(12.5 \%)$ & \\
\hline other cancers & $3(33.3 \%)$ & $0(0 \%)$ & \\
\hline Initial ICI treatment line & & & 1.09 \\
\hline $1 \mathrm{st}$ & $6(66.7 \%)$ & $5(62.5 \%)$ & \\
\hline 2nd & $1(11.1 \%)$ & $1(12.5 \%)$ & \\
\hline$\geq 3 \mathrm{rd}$ & $1(11.1 \%)$ & $2(25.0 \%)$ & \\
\hline Missing & $1(11.1 \%)$ & $0(0 \%)$ & \\
\hline Efficacy of ICls & & & 0.61 \\
\hline PR & $2(22.2 \%)$ & $3(37.5 \%)$ & \\
\hline SD & $6(66.7 \%)$ & $4(50.0 \%)$ & \\
\hline PD & $0(0 \%)$ & $1(12.5 \%)$ & \\
\hline Missing & $1(11.1 \%)$ & $0(0 \%)$ & \\
\hline ICl type & & & 1.00 \\
\hline Anti-PD-1 & $7(77.8 \%)$ & $7(87.5 \%)$ & \\
\hline Anti-PD-L1 & $2(22.2 \%)$ & $1(12.5 \%)$ & \\
\hline Number of ICl cycles & & & 0.12 \\
\hline Mean (SD) & $3.22(2.39)$ & $1.63(0.744)$ & \\
\hline Median [Min, Max] & $2.00[1.00,8.00]$ & $1.50[1.00,3.00]$ & \\
\hline Times to diagnosis & & & 0.06 \\
\hline Mean (SD) & $78.9(52.0)$ & $33.8(22.2)$ & \\
\hline Median [Min, Max] & $69.0[7.00,155]$ & $34.5[4.00,57.0]$ & \\
\hline
\end{tabular}




\subsection{Laboratory and other relevant examination results}

Baseline laboratory results were available in the majority of our patients. Troponin was elevated in all of the patients. Troponin values in most patients did not return to normal after six months and the levels fluctuate considerably during therapy. The median troponin was significantly higher in the severe myocarditis group than patients with mild myocarditis $(61.2 \mathrm{ng} / \mathrm{L}$ vs. $440 \mathrm{ng} / \mathrm{L}$, respectively, $p=0.0057)$. B-type natriuretic peptide (BNP), creatine kinase-MB (CK-MB) and myoglobin in the severe cohort were also higher in comparison to the mild cohort, but the difference was not statistically significant. $A$ significant elevation in lactate dehydrogenase (LDH) was observed between those two groups $(p=0.04)$. The median value of LDH was $233 \mathrm{IU} / \mathrm{L}$ and $519 \mathrm{IU} / \mathrm{L}$ in the mild and severe myocarditis group, respectively. White blood cells (WBC), neutrophils, lymphocytes, eosinophils, and monocytes were higher in the severe group compared with the mild group, but those parameters were not statistically significant (Table 3). CD3, CD8, and CD4 serum levels were also examined prior to ICI therapy, but no significant differences were seen. Blood lipid levels, including total cholesterol (TC), triglycerides (TG), low-density lipoprotein cholesterol (LDL-C), and high-density lipoprotein cholesterol (HDL-C) were also measured in our study. No statistical significance was detected between the severity of myocarditis and blood lipid levels. Higher TC and LDL-C levels and lower HDL-C levels were found in severe group, large-scale studies considering the relationship are needed to further explore. 
Table 3

The lab result, cardiac markers and ECG in all patients.

WBC* $10^{9} / \mathrm{L}$

Mean (SD)

Median [Min, Max]

Neutrophils $* 10^{9} / \mathrm{L}$

Mean (SD)

Median [Min, Max]

Lymphocytes* $10^{9} / \mathrm{L}$

Mean (SD)

$1.19(0.70)$

Median [Min, Max]

$0.91[0.31,2.37]$

$1.17(0.69)$

$0.90[0.43,2.37]$
Overall

$(\mathrm{N}=18)$
Mild group

$(\mathrm{N}=12)$
Severe group

$(\mathrm{N}=6)$
4.94 (1.42)

$4.70[2.52,7.77]$

$5.77[4.53,8.79]$

$P$ value

0.15

5.37 (1.65)

$3.51(1.86)$

3.07 (1.69)

4.38 (2.03)

$3.20[1.20,8.10]$

$2.81[1.20,6.13]$

$3.39[2.85,8.10]$

\section{Eosinophils* $10^{9} / \mathrm{L}$}

Mean (SD)

$0.15(0.14)$

$0.17(0.16)$

$0.10(0.10)$

Median [Min, Max]

1.00[0, 0.48]

$1.00[0.02,0.48]$

$0.07[0,0.23]$

Monocytes $* 10^{9} / \mathrm{L}$

Mean (SD)

$0.41(0.15)$

$0.37(0.15)$

$0.49(0.12)$

Median [Min, Max]

$0.41[0.06,0.64]$

$0.40[0.06,0.52]$

$0.45[0.36,0.64]$

LDH (IU/L)

0.04

Mean (SD)

485 (455)

358 (342)

740 (575)

Median [Min, Max]

$260[144,1760]$

$233[144,1350]$

$519[230,1760]$

CK-MB (ng/ml)

0.28

Mean (SD)

47.8 (76.0)

26.8 (38.2)

89.7 (115)

Median [Min, Max]

$8.30[0.89,300]$

$7.22[0.89,125]$

$44.5[1.79,300]$

Myoglobin (ng/ml)

0.14

CK-MB: Creatine kinase-MB; BNP: B-type natriuretic peptide; CRP:C-reactive protein; ECG:

Electrocardiographic;LDH:lactate dehydrogenase; NLR:neutrophil-lymphocyte ratio; TC:total cholesterol;TG:triglycerides;LDL-C:Iow-density lipoprotein cholesterol; HDL-C:high-density lipoprotein cholesterol. 


\begin{tabular}{|c|c|c|c|c|}
\hline & $\begin{array}{l}\text { Overall } \\
(\mathrm{N}=18)\end{array}$ & $\begin{array}{l}\text { Mild group } \\
(\mathrm{N}=12)\end{array}$ & $\begin{array}{l}\text { Severe group } \\
(\mathrm{N}=6)\end{array}$ & $P$ value \\
\hline Mean (SD) & 688 (912) & $505(751)$ & $1090(1160)$ & \\
\hline Median [Min, Max] & $246[32.7,3000]$ & $188[32.7,2660]$ & $902[123,3000]$ & \\
\hline Missing & $2(11.1 \%)$ & $1(8.3 \%)$ & $1(16.7 \%)$ & \\
\hline Troponin (ng/L) & & & & 0.0057 \\
\hline Mean (SD) & 359 (554) & 134 (195) & 810 (772) & \\
\hline Median [Min, Max] & $111[22.5,1990]$ & $61.2[22.5,715]$ & $440[130,1990]$ & \\
\hline BNP (ng/L) & & & & 0.34 \\
\hline Mean (SD) & $2510(6980)$ & $774(1340)$ & $5700(11600)$ & \\
\hline Median [Min, Max] & $312[19.0,29200]$ & $212[19.0,4620]$ & $933[85.0,29200]$ & \\
\hline Missing & $1(5.6 \%)$ & $1(8.3 \%)$ & $0(0 \%)$ & \\
\hline РCT (ng/ml) & & & & 0.87 \\
\hline Mean (SD) & $1.07(1.54)$ & $0.78(0.960)$ & $1.48(2.18)$ & \\
\hline Median [Min, Max] & $0.18[0.02,5.02]$ & $0.22[0.02,2.12]$ & $0.13[0.03,5.02]$ & \\
\hline Missing & $6(33.3 \%)$ & $5(41.7 \%)$ & $1(16.7 \%)$ & \\
\hline IL-6 (pg/ml) & & & & 0.92 \\
\hline Mean (SD) & $265(805)$ & $401(1010)$ & $26.3(32.4)$ & \\
\hline Median [Min, Max] & $12.8[2.29,2690]$ & $12.8[2.29,2690]$ & $12.6[2.77,77.1]$ & \\
\hline Missing & 7 (38.9\%) & $5(41.7 \%)$ & $2(33.3 \%)$ & \\
\hline CRP (mg/L) & & & & 0.74 \\
\hline Mean (SD) & $45.6(42.4)$ & $53.1(49.5)$ & $34.9(32.0)$ & \\
\hline Median [Min, Max] & $42.7[2.97,131]$ & $68.5[2.97,131]$ & $22.2[4.75,74.9]$ & \\
\hline Missing & $6(33.3 \%)$ & $5(41.7 \%)$ & $1(16.7 \%)$ & \\
\hline NLR & & & & 0.54 \\
\hline Mean (SD) & $5.00(6.05)$ & $4.10(3.56)$ & $6.81(9.51)$ & \\
\hline
\end{tabular}

CK-MB: Creatine kinase-MB; BNP: B-type natriuretic peptide; CRP:C-reactive protein; ECG: Electrocardiographic;LDH:lactate dehydrogenase; NLR:neutrophil-lymphocyte ratio; TC:total cholesterol;TG:triglycerides;LD L-C:Iow-density lipoprotein cholesterol; HDL-C:high-density lipoprotein cholesterol. 


\begin{tabular}{|c|c|c|c|c|}
\hline & $\begin{array}{l}\text { Overall } \\
(\mathrm{N}=18)\end{array}$ & $\begin{array}{l}\text { Mild group } \\
(\mathrm{N}=12)\end{array}$ & $\begin{array}{l}\text { Severe group } \\
(\mathrm{N}=6)\end{array}$ & $P$ value \\
\hline Median [Min, Max] & $3.04[0.51,26.1]$ & $2.71[0.51,10.6]$ & $3.09[1.59,26.1]$ & \\
\hline CD3 & & & & 0.76 \\
\hline Mean (SD) & $64.3(16.0)$ & $66.0(19.2)$ & $62.0(11.6)$ & \\
\hline Median [Min, Max] & $63.6[39.6,90.1]$ & $64.7[39.6,90.1]$ & $62.4[46.6,76.7]$ & \\
\hline CD4 & & & & 0.53 \\
\hline Mean (SD) & $28.7(12.1)$ & $31.7(14.3)$ & $24.5(7.6)$ & \\
\hline Median [Min, Max] & $27.1[16.1,58.3]$ & $29.5[16.1,58.3]$ & $22.1[17.1,35.8]$ & \\
\hline CD8 & & & & 0.64 \\
\hline Mean (SD) & $31.3(15.2)$ & $30.6(17.7)$ & $32.2(12.9)$ & \\
\hline Median [Min, Max] & $27.5[17.0,68.6]$ & $27.4[17.0,68.6]$ & $31.3[17.7,49.4]$ & \\
\hline \multicolumn{5}{|l|}{ Blood lipid levels } \\
\hline $\mathrm{TC}(\mathrm{mmol} / \mathrm{L})$ & $4.44(0.876)$ & $4.39(1.02)$ & $4.53(0.569)$ & 0.49 \\
\hline $\mathrm{TG}(\mathrm{mmol} / \mathrm{L})$ & $1.73(0.702)$ & $1.77(0.744)$ & $1.66(0.671)$ & 0.82 \\
\hline $\mathrm{HDL}-\mathrm{C}(\mathrm{mmol} / \mathrm{L})$ & $1.08(0.302)$ & $1.19(0.227)$ & $0.857(0.328)$ & 0.06 \\
\hline LDL-C(mmol/L) & $2.69(0.783)$ & $2.52(0.904)$ & $3.02(0.302)$ & 0.06 \\
\hline \multicolumn{5}{|l|}{ ECG } \\
\hline abnormal T wave & $7 / 18$ & $4 / 12$ & $3 / 6$ & 0.63 \\
\hline abnormal ST segment & $3 / 18$ & $3 / 12$ & $0 / 6$ & 0.52 \\
\hline conduction defects & $3 / 18$ & $2 / 12$ & $1 / 6$ & 1.00 \\
\hline Sinus tachycardia & $3 / 18$ & $2 / 12$ & $1 / 6$ & 1.00 \\
\hline Normal ECG & $1 / 18$ & $1 / 12$ & $0 / 6$ & - \\
\hline \multicolumn{5}{|c|}{$\begin{array}{l}\text { CK-MB: Creatine kinase-MB; BNP: B-type natriuretic peptide; CRP:C-reactive protein; ECG: } \\
\text { Electrocardiographic;LDH:lactate dehydrogenase; NLR:neutrophil-lymphocyte ratio; TC:total } \\
\text { cholesterol;TG:triglycerides;LDL-C:low-density lipoprotein cholesterol; HDL-C:high-density lipoprotein } \\
\text { cholesterol. }\end{array}$} \\
\hline
\end{tabular}

Every patient received an electrocardiographic (ECG) examination, and nearly every case of myocarditis showed an irregular ECG (94.4\%). It was found that 7 cases had abnormal T waves, 3 cases had an abnormal ST segment, and conduction defects were also found in 3 patients. Additionally, sinus 
tachycardia showed in three cases. But such difference in ECG between those two groups did not have statistically significant.

Echocardiography was performed in 17 patients. Two patients were discovered to have a ventricular wall movement disorder, and two patients had left ventricular systolic dysfunction (LVEF $\leq 50 \%$ ). One patient was diagnosed with severe myocarditis and third-degree atrioventricular block, with an LVEF of $40 \%$. Coronary angiography was also performed in this patient which revealed the anterior descending branch, circumflex branch and right coronary artery were stenosed and the first diagonal branch had the most severe stenosis of nearly $40 \%$. However, the patient had no history of coronary heart disease. Coronary angiography results were also obtained from the other four patients. One patient in the severe group had stenosis in the left anterior descending branch, circumflex branch and right coronary artery. The diagnosis of myocarditis in these 2 cases was made by a multidisciplinary team and they all matched with the inclusion criteria. Nevertheless, there are no meaningful findings in the other three patients in the mild group. A cardiac magnetic resonance imaging (MRI) investigation was performed in six cases, however, we were unable to find meaningful data in five of them. Only one patient showed myocardial edema on T2-weighted imaging. Furthermore, one patient with severe myocarditis received cardiac 18Ffluorodeoxyglucose (18F-FDG) position emission tomography magnetic resonance imaging (PET-MRI) which revealed enhanced FDG metabolism at the left ventricle. All patients were treated with steroids once myocarditis was diagnosed. The initial steroid dose was an equivalent of methylprednisolone (1-2 $\mathrm{mg} / \mathrm{kg}$ ), which was sustained for 3-5days and followed by a long-term oral steroid taper. In four patients, intravenous gammaglobulin was used to relieve their symptoms. Unfortunately, one patient died as a result of myocarditis-related complications.

\subsection{Concomitant irAEs and outcomes}

$66.7 \%$ of patients (12 cases) were concurrent with at least one irAE (Figure $2 \mathrm{~A}$ ). 9 patients developed another irAE: six patients were diagnosed with immune-related myositis, two acquired hypothyroidism and one suffered pneumonitis. One patient developed other two irAEs and two patients were concurrent with three irAEs. Overall, there was no difference in the prevalence between the mild and severe groups $(P>0.5)$. Myositis $(n=8)$ and hypothyroidism $(n=4)$ were the most common irAEs in our cohorts.

The difference between those who had additional irAE and those who did not was also discussed in our research (Table 4). Patients without concomitant irAEs $(\mathrm{N}=6)$ were all males and treated with PD-1 antibodies. The onset time of patients with single irAE was later than patients with multi-irAEs. Baseline characteristics were explored and identified that the alcohol history was significantly different. All patients with concomitant irAEs did not have an alcohol history $(p=0.03)$. However, this result should be validated in a larger cohort study. There were also statistically significant changes in LDH and myoglobin. Patients with multi-irAEs have a higher levels of $\mathrm{LDH}(250 \mathrm{IU} / \mathrm{L}$ v.s $603 \mathrm{IU} / \mathrm{L}, \mathrm{P}=0.02)$ and myoglobin(112 $\mathrm{ng} / \mathrm{ml}$ v.s $950 \mathrm{ng} / \mathrm{ml}, \mathrm{P}=0.02)$. There was also a trend of higher CK-MB, BNP, and a lower troponin in patients with multi-irAEs, although these changes were not statistically significant. 
Table 4

Comparison of myocarditis patients with Single irAE or Multi-irAE.

\section{Severity}

mild

Severe

Age

Gender

Male

Female

Cancer type

Other cancers

lung cancer

thymic carcinoma

ICl type

Anti-PD-1

Anti-PD-L1

Number of ICl cycles

Times to onset

WBC* $10^{9} / \mathrm{L}$

Neutrophils* $10^{9} / \mathrm{L}$

Lymphocytes* $10^{9} / \mathrm{L}$

Monocytes ${ }^{*} 10^{9} / \mathrm{L}$

Eosinophils $* 10^{9} / \mathrm{L}$

NLR

CK-MB (ng/ml)

Myoglobin (ng/ml)

$0(0.00 \%)$

$2(33.3 \%)$

$4(66.7 \%)$

$0(0.00 \%)$

$6(100 \%)$

$0(0.00 \%)$

$3.33(2.50)$

72.0 (50.7)

$4.86(0.99)$

$3.26(0.70)$

$1.07(0.65)$

$0.40(0.26)$

$0.11(0.09)$

$4.25(3.00)$

$8.60(10.4)$

$112(65.0)$

\section{Single irAE $(N=6)$}

$4(66.7 \%)$

$2(33.3 \%)$

65.7 (10.5)

Multi-irAE(N=12)

$P$ value

$8(66.7 \%)$

$4(33.3 \%)$

53.8 (16.0)

0.12

0.52

$9(75.0 \%)$

3 (25.0\%)

0.42

2 (16.7\%)

6 (50.0\%)

$4(33.3 \%)$

0.52

9 (75.0\%)

3 (25.0\%)

1.92 (1.44)

0.12

46.8 (42.8)

0.28

5.63 (1.89)

0.28

3.64 (2.25)

0.74

$1.24(0.74)$

0.85

$0.42(0.06)$

0.48

$0.17(0.16)$

0.74

5.38 (7.21)

0.67

67.3 (87.3)

0.08

950 (1003)

0.02

${ }^{*}$ Data are shown as means \pm SD. CK-MB: Creatine kinase-MB; BNP: B-type natriuretic peptide; CRP:Creactive protein; ECG: Electrocardiographic; LDH: lactate dehydrogenase; NLR: neutrophil-lymphocyte ratio. 


\begin{tabular}{|c|c|c|c|}
\hline & Single irAE $(N=6)$ & Multi-irAE(N=12) & $P$ value \\
\hline Troponin (ng/L) & 479 (763) & $299(445)$ & 0.89 \\
\hline BNP (ng/L) & $992(1042)$ & 3341 (8679) & 0.72 \\
\hline РCT (ng/ml) & $2.45(2.42)$ & $0.61(0.91)$ & 0.12 \\
\hline CRP (mg/L) & $30.6(42.1)$ & $50.5(43.7)$ & 0.64 \\
\hline LDH (IU/L) & 250 (107) & 603 (519) & 0.02 \\
\hline IL-6 (pg/ml) & 1351 (1893) & $23.2(30.1)$ & 0.34 \\
\hline CD3 & $65.3(17.6)$ & $64.0(16.5)$ & 1.00 \\
\hline CD4 & $40.0(16.6)$ & $24.9(8.26)$ & 0.15 \\
\hline CD8 & $22.2(7.91)$ & $34.3(16.2)$ & 0.21 \\
\hline
\end{tabular}

\subsection{Comparison of the baseline levels of serum myocardial enzymes and LDH between different groups.}

Our results revealed that the serum myocardial enzymes and LDH may play an suggestive role in the early diagnosis of immune-related myocardial injury. But there was no statistical difference of baseline levels of those markers between patients with severe myocarditis and mild myocarditis $(P>0.05$,Table 5$)$. To further exploring the difference of those marker between patients with and without myocarditis, we retrospectively screened 30 patients without myocarditis in our hospital who treated with ICls. There was no statistical significance on the differences in serum myocardial enzymes and LDH between patients who experienced immune-related myocarditis group and who did not experienced ( $P>0.05$, Figure $2 B)$.We also analyzed the changes of those markers during treated with ICls in patients without immuneassociated myocarditis. The comparison of LDH, troponin, CK-MB,BNP and myoglobin levels between the baseline levels and after received one or two cycles of ICls were not statistically significant $(P>0.05)$. 
Table 5

Comparison of the baseline levels of serum myocardial enzymes and LDH between different groups

\begin{tabular}{|llll|}
\hline & Mild group & Severe group & P value \\
\hline Troponin $(\mathrm{ng} / \mathrm{L})$ & $12.9(8.52)$ & $8.58(3.79)$ & 0.67 \\
\hline Myoglobin $(\mathrm{ng} / \mathrm{ml})$ & $31.3(13.2)$ & $24.1(3.61)$ & 0.71 \\
\hline CK-MB $(\mathrm{ng} / \mathrm{ml})$ & $1.49(1.12)$ & $1.54(0.546)$ & 0.57 \\
\hline BNP $(\mathrm{ng} / \mathrm{L})$ & $94.5(57.5)$ & $59.0(43.8)$ & 0.67 \\
\hline LDH $(\mathrm{IU} / \mathrm{L})$ & $221(99.5)$ & $179(29.2)$ & 0.34 \\
\hline
\end{tabular}

\subsection{The role of troponin and LDH levels in diagnosing severe myocarditis.}

To confirm our findings, we searched PubMed for relevant articles and selected 30 for analysis.. The results are coincident with ours. Patients with severe myocarditis have a higher troponin $(p=0.005)$ and an earlier onset time (19.1 v.s 29.7 days, $p=0.014$ ) compared with the mild group. In addition, significant difference was found in the outcome between two groups (Table 6). Patients in the severe group have a higher mortality ( $53.3 \%$ v.s $6.7 \%, p=0.041)$. The LDH in most studies were not eligible and thus we did not analyzed. 
Table 6

Patients with immune-related myocarditis searched in Pubmed were analysed.

\begin{tabular}{|c|c|c|c|}
\hline & Mild group $(\mathrm{N}=15)$ & Severe group $(\mathrm{N}=15)$ & $P$ value \\
\hline Number of ICl cycles & & & 0.64 \\
\hline Mean (SD) & $1.53(1.06)$ & $1.27(0.458)$ & \\
\hline Median [Min, Max] & $1.00[1.00,5.00]$ & $1.00[1.00,2.00]$ & \\
\hline Times to diagnosis & & & 0.041 \\
\hline Mean (SD) & $29.7(20.8)$ & $19.1(9.38)$ & \\
\hline Median [Min, Max] & $28.0[4.00,90.0]$ & $19.0[4.00,45.0]$ & \\
\hline Troponin (ng/L) & & & 0.005 \\
\hline Mean (SD) & $1200(1280)$ & $4540(4580)$ & \\
\hline Median [Min, Max] & $1030[44.0,3720]$ & $3230[806,16300]$ & \\
\hline Outcomes & & & 0.014 \\
\hline Lived & $14(93.3 \%)$ & $7(46.7 \%)$ & \\
\hline Died & $1(6.7 \%)$ & $8(53.3 \%)$ & \\
\hline
\end{tabular}

\section{Discussion}

Immune checkpoint inhibitors have drastically improved clinical outcomes and they are increasingly being licensed for use in the early stage of cancer and combination with other anti-tumor therapies such as chemotherapy or targeted therapy ${ }^{1}$. However, due to the increased usage of these medications, immune-related adverse events in patients receiving ICls are becoming more widely recognized, which may limit their clinical applicability. One of the most fatal irAEs is immune-related myocarditis. Due to its severity and high mortality, there is a growing interest in the further study ${ }^{9}$. The risk of myocarditis varies depending on the treatment regimen and the $\mathrm{ICl}$ drugs used. Overall, ICl-related myocarditis was reported to be around $1 \%$ of the incidence. Previous research had shown that dual ICls regimens caused a higher incidence than monotherapy ${ }^{10}$. The incidence of monotherapy, dual ICls therapies and ICl plus chemotherapy were all found to be $3.1 \%, 5.8 \%$, and $3.7 \%$, respectively. There were also variations in the occurrence of myocarditis among the various classes of $\mathrm{ICls}^{8}$. Myocarditis is more likely to be caused by the CTLA-4 antibody. Furthermore, it has been observed that nivolumab (anti-PD-1) had a decreased incidence of cardiac irAEs ${ }^{11}$. The underlying mechanism still unclear. T-cell infiltration into the myocardium, increased auto-antibodies acting on self-antigens, and increased $T$ cells reacting to antigens shared by cancer and normal cells could all be contributors to the pathomechanism of the irAEs 12. 
There is a scarcity of information on this potentially fatal adverse event. Our current understanding of the illness is limited, and patient-derived evidence is scarce. Recent research has concentrated on examining the disease's overall characteristics while neglecting the individual variabilities. The goal of this retrospective study was to provide new insights about such a rare irAE and explore risk factors for severe myocarditis and multi-irAEs. According to Common Terminology Criteria for Adverse Events (CTCAE), AE grading is currently based on the outcomes of biomarkers, ECG, symptoms and cardiac complications ${ }^{13}$. Patients with grade 1 need to be closely monitored during therapy. The patient in grade 1 is asymptomatic but has aberrant cardiac biomarkers with an irregular ECG. Compared to grade 1, patients with grade 2 have minor symptoms. Symptoms in grade 3 patients are more severe and required the use of steroids to manage. Grade 4 is defined as moderate to severe decompensation of life-threatening situations that demand intravenous injection of medication or intervention. In our study, patients were divided into groups based on their clinical manifestations and whether they had haemodynamic complications such as heart failure, cardiogenic shock, or arrhythmia, which may be more useful in assisting clinicians in determining a patient's condition and prognosis. Patients in severe group were categorized as grade 3-4 toxicity and in the mild group were classified as grade 1-2 toxicity.

It is of critical importance to distinguish between mild and severe myocarditis due to the high mortality rate. Several studies have identified treatment regimens, comorbidities such as hypertension and diabetes, and tobacco use as risk factors for cardiotoxicity ${ }^{14}$. However, Subgroup analyses based on age, gender, tobacco usage, diabetes, and cancer stage revealed no differences in our study. According to our findings, hypertension and cancer type may not correlate to the severity of myocarditis. Contrary to earlier reports, our data have shown that none of the patients in the severe group had hypertension. In addition, none of the 18 patients had coronary artery disease or any other type of cardiac disease. No statistical significance was detected between the severity of myocarditis and blood lipid levels.

The most common malignancy type in the mild group was lung cancer, while the most common type in the severe group was thymic carcinoma. The fundamental cause of the discrepancy in our study was unknown. This could be due to the fact that a large percentage of patients with thymic cancer have an autoimmune syndrome, which could be an independent risk factor for ICl-associated cardiotoxicity. The thymus plays an essential role in the development of T-cells. Although autoimmune disease patients are not the focus of our research, the function and composition of T-cells, which are important components of the immune system, are similarly aberrant in the thymoma microenvironment ${ }^{15}$. Therefore, it is critical to keep track of the occurrence of myocarditis in thymic carcinoma patients receiving ICl.

According to the literature, immune-related myocarditis frequently develops soon after starting $\mathrm{ICI}$ therapy. The median time of onset was 34 days, with the majority of cases occurring within three months 8 . In our studies, the median length was 2 cycles and the onset time was 51 days (range: $4-155$ ), which is longer than previous reports ${ }^{16}$. Patients with severe myocarditis had a faster onset time than those with mild myocarditis. Myocarditis is more likely to present at an early age in patients in the mild group who were treated with combination therapy, although the results of onset time were not statistically 
significant. Treatment lines, ICl type, number of $\mathrm{ICl}$ cycles, and ICI efficacy all had no statistically significant differences. Recent research suggested that irAEs have been linked to a long-term response and therapeutic benefit ${ }^{16-18}$. Among the 16 patients, 5 patients had a partial response (PR), 10 patients had stable disease (SD), and 1 patient had progressive disease (PD).

Early identification of severe myocarditis followed by timely treatment is essential to reduce mortality and assisting physicians in personalized medicine decision-making. Our study suggested that the troponin and LDH might contribute to recognize the severe myocarditis as soon as possible. Previous studies have demonstrated that monitoring troponin during treatment is reasonable $e^{6,19,20}$, but the relationship between LDH and cardiotoxicity were never reported. Patients with severe myocarditis are more likely have a higher LDH. Furthermore, real-time screening of concomitant irAE is essential in patient experiencing immune-related myocarditis, especially in patient with higher LDH and myoglobin levels. In addition, the level of troponin also correlated with outcomes.

The diagnosis and management of this disorder continue to be a clinical and research challenge. Myocardial biopsy is the gold standard for diagnosis. However, the complications may have an adverse effect on clinical utility and outcomes ${ }^{21}$. The relevance of cardiac MRI, FDG-PET, and coronary angiography in the identification of myocarditis has also been highlighted in recent research ${ }^{22-24}$. However, performing those examinations may miss the optimal treatment time and result in negative repercussions. A multidisciplinary team (MDT) approach is crucial for assessing suspected immunerelated myocarditis, since it aids decision-making and lowers death rates. The mortality rate was reported to be as high as $46 \%{ }^{25}$, while in our study was $5.6 \%$ (1/18). Early identification by MDT and the use of steroids may have contributed to the lower mortality rate. For severe myocarditis, there is no question that corticosteroids should be used, but the treatment of mild myocarditis by steroids is still unknown and requires further research. Our study provides clinical relevance and rationale for initiating corticosteroid therapy regardless of the severity of myocarditis. All 18 patients in our study received steroid treatment. An equivalent dose of (methyl)prednisolone (1-2 mg/ $\mathrm{kg}$ ) is given for the first 3-5 days, followed by a long-term oral steroid taper. In addition to the use of steroids, intravenous gammaglobulin should be considered to alleviate the symptoms of patients based on clinical need. After being diagnosed with myocarditis, no one disputes ICls again.

Our research fills a gap in the literature and sheds new light on a rare irAE. However, there are a few flaws worth mentioning. It's worth noting the inherent bias in any single-institution retrospective analysis, and also the limited sample population in our study. Due to the challenges in diagnosis, the vast majority of myocarditis events may be overlooked or misdiagnosed. Furthermore, extreme vigilance is essential in the therapy and the avoidance of excessive corticosteroid usage for bias results. Further investigation is required as an outcome of our findings.

\section{Declarations}


This work was supported by the National Natural Science Foundation of China (Grant No.81572850) and 1.3.5 Project for Disciplines of Excellence, West China Hospital, Sichuan University (Grant No. ZYJC21043).

\section{Conflict of Interest Statement}

All authors declare no conflicts of interest in relation to this work.

\section{Data availability statement}

All data relevant to the study are available from the corresponding author on reasonable request.

\section{Author contributions}

ML: Conceptualization, Supervision and Data curation; YL: Writing original draft, Formal analysis, and Validation; Other authors: Supervision and Validation

\section{References}

1. Postow MA, Callahan MK, Wolchok JD. Immune Checkpoint Blockade in Cancer Therapy. Journal of Clinical Oncology 2015;33:1974-82.

2. Gong J, Chehrazi-Raffle A, Reddi S, Salgia R. Development of PD-1 and PD-L1 inhibitors as a form of cancer immunotherapy: a comprehensive review of registration trials and future considerations. $\mathrm{J}$ Immunother Cancer 2018;6:8.

3. Pardoll DM. The blockade of immune checkpoints in cancer immunotherapy. Nat Rev Cancer 2012;12:252-64.

4. Zhu H, Ivanovic M, Nguyen A, Nguyen PK, Wu SM. Immune checkpoint inhibitor cardiotoxicity: Breaking barriers in the cardiovascular immune landscape. J Mol Cell Cardiol 2021;160:121-27.

5. Escudier M, Cautela J, Malissen N, Ancedy Y, Orabona M, Pinto J, Monestier S, Grob J-J, Scemama U, Jacquier A, Lalevee N, Barraud J, et al. Clinical Features, Management, and Outcomes of Immune Checkpoint Inhibitor-Related Cardiotoxicity. Circulation 2017;136:2085-87.

6. Liu Q, Yu Y, Lin J, Wang Y, Ai L, Li Q, Wu W, Jin H, Li S, Liu M, Yu S, Liu T. Treatment strategy for myocarditis in patients using immune checkpoint inhibitors or combined anti-vascular endothelial growth factor therapy by clinical severity. Eur J Cancer 2021;157:10-20.

7. Moslehi JJ, Salem J-E, Sosman JA, Lebrun-Vignes B, Johnson DB. Increased reporting of fatal immune checkpoint inhibitor-associated myocarditis. Lancet 2018;391:933.

8. Mahmood SS, Fradley MG, Cohen JV, Nohria A, Reynolds KL, Heinzerling LM, Sullivan RJ, Damrongwatanasuk R, Chen CL, Gupta D, Kirchberger MC, Awadalla M, et al. Myocarditis in Patients Treated With Immune Checkpoint Inhibitors. J Am Coll Cardiol 2018;71:1755-64.

9. Ganatra S, Neilan TG. Immune Checkpoint Inhibitor-Associated Myocarditis. Oncologist 2018;23:87986. 
10. Rubio-Infante N, Ramírez-Flores YA, Castillo EC, Lozano O, García-Rivas G, Torre-Amione G. Cardiotoxicity associated with immune checkpoint inhibitor therapy: a meta-analysis. Eur J Heart Fail 2021.

11. Mascolo A, Scavone C, Ferrajolo C, Rafaniello C, Danesi R, Del Re M, Russo A, Coscioni E, Rossi F, Alfano R, Capuano A. Immune Checkpoint Inhibitors and Cardiotoxicity: An Analysis of Spontaneous Reports in Eudravigilance. Drug safety 2021.

12. Baik AH, Tsai KK, Oh DY, Aras MA. Mechanisms and clinical manifestations of cardiovascular toxicities associated with immune checkpoint inhibitors. Clinical Science 2021;135:703-24.

13. Brahmer JR, Lacchetti C, Schneider BJ, Atkins MB, Brassil KJ, Caterino JM, Chau I, Ernstoff MS, Gardner JM, Ginex P, Hallmeyer S, Holter Chakrabarty J, et al. Management of Immune-Related Adverse Events in Patients Treated With Immune Checkpoint Inhibitor Therapy: American Society of Clinical Oncology Clinical Practice Guideline. Journal of clinical oncology : official journal of the American Society of Clinical Oncology 2018;36:1714-68.

14. Patel RP, Parikh R, Gunturu KS, Tariq RZ, Dani SS, Ganatra S, Nohria A. Cardiotoxicity of Immune Checkpoint Inhibitors. Curr Oncol Rep 2021;23:79.

15. Hung C-T, Tsai T-F, Chen J-S, Hsieh M-S. Thymoma-associated multiorgan autoimmunity. BMJ case reports 2019;12.

16. Ma R, Wang Q, Meng D, Li K, Zhang Y. Immune checkpoint inhibitors-related myocarditis in patients with cancer: an analysis of international spontaneous reporting systems. BMC cancer 2021;21:38.

17. Paderi A, Giorgione R, Giommoni E, Mela M, Rossi V, Doni L, Minervini A, Carini M, Pillozzi S, Antonuzzo L. Association between Immune Related Adverse Events and Outcome in Patients with Metastatic Renal Cell Carcinoma Treated with Immune Checkpoint Inhibitors. Cancers 2021;13.

18. Dimitriou F, Staeger R, Ak M, Maissen M, Kudura K, Barysch M, Levesque M, Cheng P, Dummer R, Mangana J. Frequency, Treatment and Outcome of Immune-Related Toxicities in Patients with Immune-Checkpoint Inhibitors for Advanced Melanoma: Results from an Institutional Database Analysis. Cancers 2021;13.

19. Sarocchi M, Grossi F, Arboscello E, Bellodi A, Genova C, Dal Bello MG, Rijavec E, Barletta G, Rossi G, Biello F, Ghigliotti G, Canepa M, et al. Serial Troponin for Early Detection of Nivolumab Cardiotoxicity in Advanced Non-Small Cell Lung Cancer Patients. Oncologist 2018;23:936-42.

20. Puzanov I, Subramanian P, Yatsynovich YV, Jacobs DM, Chilbert MR, Sharma UC, Ito F, Feuerstein SG, Stefanovic F, Switzer B, Hicar MD, Curtis AB, et al. Clinical characteristics, time course, treatment and outcomes of patients with immune checkpoint inhibitor-associated myocarditis. J Immunother Cancer 2021;9.

21. Imran M, Wang L, McCrohon J, Yu C, Holloway C, Otton J, Huang J, Stehning C, Moffat K, Ross J, Puntmann V, Vassiliou V, et al. Native T Mapping in the Diagnosis of Cardiac Allograft Rejection: A Prospective Histologically Validated Study. JACC. Cardiovascular imaging 2019;12:1618-28.

22. Ederhy S, Salem J, Dercle L, Hasan A, Chauvet-Droit M, Nhan P, Ammari S, Pinna B, Redheuil A, Boussouar S, Champiat S, Soulat-Dufour L, et al. Role of Cardiac Imaging in the Diagnosis of 
Immune Checkpoints Inhibitors Related Myocarditis. Front Oncol 2021;11:640985.

23. Spallarossa P, Sarocchi M, Tini G, Arboscello E, Toma M, Ameri P, Porto I. How to Monitor Cardiac Complications of Immune Checkpoint Inhibitor Therapy. Frontiers in pharmacology 2020;11:972.

24. Nensa F, Kloth J, Tezgah E, Poeppel TD, Heusch P, Goebel J, Nassenstein K, Schlosser T. Feasibility of FDG-PET in myocarditis: Comparison to CMR using integrated PET/MRI. J Nucl Cardiol 2018;25:78594.

25. Mahmood S, Fradley M, Cohen J, Nohria A, Reynolds K, Heinzerling L, Sullivan R, Damrongwatanasuk R, Chen C, Gupta D, Kirchberger M, Awadalla M, et al. Myocarditis in Patients Treated With Immune Checkpoint Inhibitors. J Am Coll Cardiol 2018;71:1755-64.

\section{Figures}

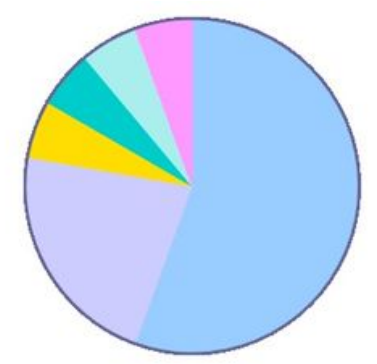

All cases $18(\mathrm{~N}=18)$

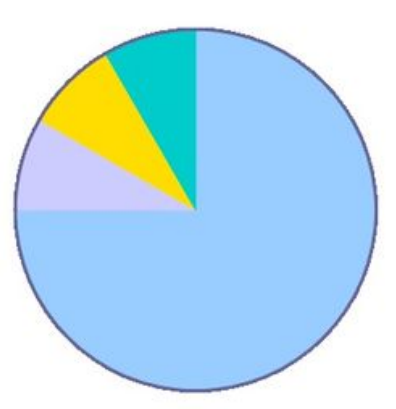

Mild myocarditis $\mathrm{N}=12$
Lung cancer $(75.0 \%)$

$\square$ Thymic carcinoma $(8.33 \%)$

Head and neck cancer $(8.33 \%)$

$\square$ Bladder cancer (8.33\%)

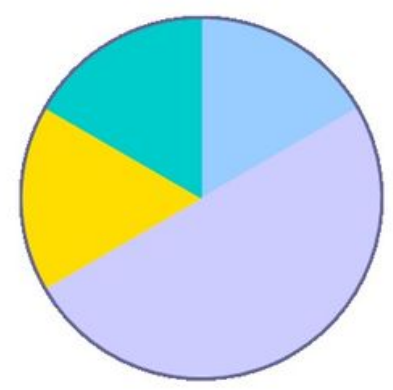

Severe myocarditis $\mathrm{N}=6$ $\square$ Lung cancer $(16.67 \%)$

$\square$ Thymic carcinoma $(50.00 \%)$

- Sigmoid colon cancer (16.67\%)

$\square$ Hepatocellular carcinoma $(16.67 \%)$

\section{Figure 1}

Cancer type distribution of patients with mild or severe Myocarditis. 
A
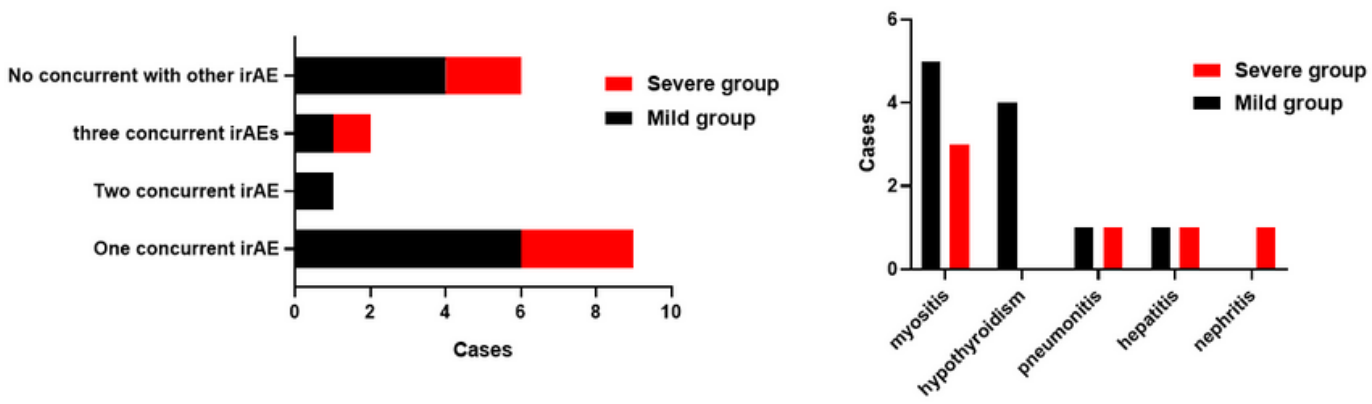

B
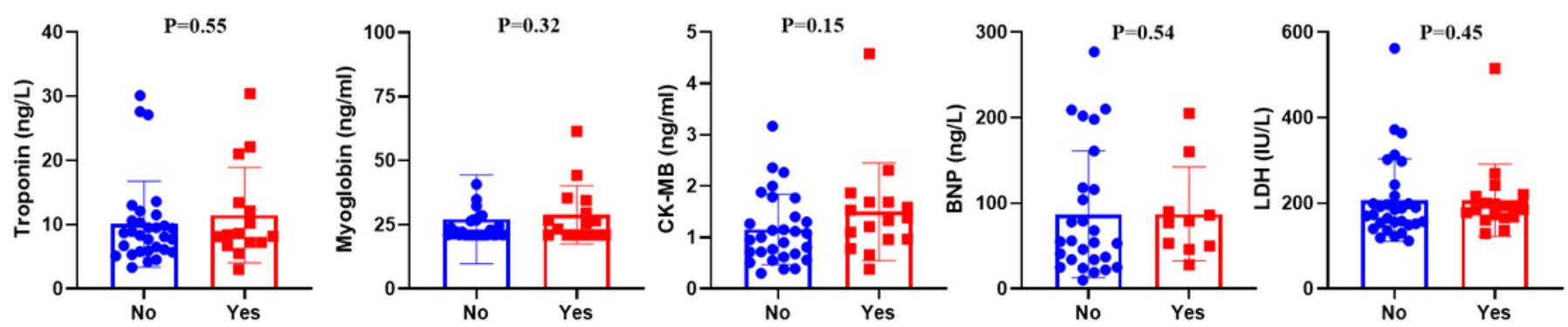

Figure 2

A: The distribution of concurrent irAEs in our study. B: Serum myocardial enzymes and LDH between patients with or without immune-related myocarditis. 